\title{
Why should the molecular characterization of inflammasome-induced exosomal cargo be done?
}

\author{
Atilla Engin ${ }^{1}$, Ayse Basak Engin ${ }^{2}$ \\ ${ }^{1}$ Department of General Surgery, Faculty of Medicine, Gazi University, Ankara, Turkey; ${ }^{2}$ Department of Toxicology, Faculty of Pharmacy, Gazi \\ University, Ankara, Turkey \\ Correspondence to: Atilla Engin. Department of General Surgery, Faculty of Medicine, Gazi University, Ankara, Turkey. Email: dr.aengin@gmail.com. \\ Comment on: Budden CF, Gearing LJ, Kaiser R, et al. Inflammasome-induced extracellular vesicles harbour distinct RNA signatures and alter \\ bystander macrophage responses. J Extracell Vesicles 2021;10:e12127.
}

Received: 11 September 2021; Accepted: 22 October 2021; Published: 30 November 2021.

doi: $10.21037 /$ exrna-21-16

View this article at: https://dx.doi.org/10.21037/exrna-21-16

Exosomes (EXSs) are nano-sized and circulating membrane-bound extracellular vesicles (EVs) which protect the stability of their cargo, including messenger ribonucleic acid (mRNA), microRNA (miRNA) and other proteins from degradation. Upon taken up by recipient cells, they deliver their cargo molecules to the target cells. When inflammatory stimuli activate innate immune signaling receptors, cargo molecules modify the molecules of recipient cell. Because molecules carried with EXS-cargo are thought to be biomarkers, the role of EXSs is emerging as an important new area of biomedical research.

In addition to inflammatory mediators, EXSs, which are secreted by macrophages play pivotal roles in intercellular communications and multi biological functions (1). The immune response-related proteins and signaling pathways are specifically enriched in inflammasome-derived EXSs. This type of EXSs directly activates nuclear factor kappa B $(\mathrm{NF}-\kappa \mathrm{B})$ signaling pathway and expresses pro-interleukin 1-beta (pro-IL-1 $\beta$ ) and pro-IL-18 via its transcriptional upregulation (2).

EXSs as potential carriers for targeted gene, are thought to promote inflammation via releasing of inflammasome components during the inflammatory response. Of note, there is a need for further studies to elucidate the role of EXSs by using improved detection and characterization methods (3). Indeed, inflammasomes are critical for both local and systemic inflammation. Canonical type NODlike receptor protein 3 (NLRP3) inflammasome is known to sense multiple microbial and endogenous danger signals, and its activation results in caspase-1, IL- $1 \beta$ and IL-18, dependent processes. However, non-canonical, caspase4/5-dependent inflammasomes bind to intracellular lipopolysaccharides (LPS), and directly regulate pyroptosis $(4,5)$. Contrarily, Budden et al. have proposed that the pathways eliciting caspase 1-driven cell death strongly induce EXS release, whereas stimuli, which do not induce pyroptosis are weaker stimuli for EXS release (6). In this context two noteworthy results are emerged in this study. EXSs released from inflammasome-activated macrophages have a specific RNA signature and contain interferonbeta (IFN- $\beta$ ). IFN- $\beta$ is responsible for the induction of IFN-stimulated genes (ISGs) in EXS recipient cells, independently of NLRP3 expression. Thereby, this cargo induces an IFN signature in bystander cells and leads to silencing of NLRP3 inflammasome response (6). In this situation, IFN- $\beta$ inhibits NLRP 3 inflammasomes in a signal transducer and activator of transcription 1 (STAT1)dependent manner, while decreases the amounts of pro-IL$1 \beta$ in a STAT3-dependent manner (7). In case of toll-like receptor (TLR) activation, Toll/IL-1 receptor homology (TIR)-domain-containing adaptor protein-inducing IFN- $\beta$ (TRIF) interacts with receptor-interacting protein 1 (RIP1), which is responsible for the activation of NF$\kappa \mathrm{B}$. Thus, stimulation with TLR ligands induces type I IFN production in addition to proinflammatory signals via TRIF-dependent pathway (8). However, activation of inflammasome controls the post-translational proteolytic activity of IL-1 $\beta$, IL-18, pore-forming gasdermins and caspase-1. Pyroptosis commonly occurs with the activation of the caspase-1-dependent canonical pathway and the 
caspase-4/5/11-dependent non-canonical pathway (9). In the first phase of inflammasome activation, LPS binds to TLR4. When macrophages are primed by LPS, activation of NF- $\mathrm{KB}$ signaling promotes expression of inflammasomerelated molecules, including pro-IL-1 $\beta$ and NLRP3 (10). Budden et al. showed that EXS release upon NLRP3 activation is temporally correlated with IL- $1 \beta$ release and is an NLRP3-, caspase 1-, and gasdermin D-dependent event. In this study, it is noted that the transcriptional cargo of EXSs is released by human macrophages upon stimulation with inflammasome or TLR stimuli. However, RNA content of EXS, which is induced by different NLRP3 activators defined as EXS-associated NLRP3 signature is different from the RNA content of TLR-induced EXSs (6).

To express inflammasome-related molecules, following

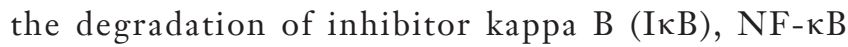
undergoes nuclear translocation and binds to the promoter regions of its downstream target genes (11). Wang et al. showed that LPS-induced expression of NLRP3 and pro-IL- $1 \beta$, is dependent on NF- $\kappa \mathrm{B}$ signaling. Thus, in connection with the transcriptional state, EXSs may inhibit IL-1 $\beta$ production and pyroptosis in macrophages by suppressing the NF- $\kappa \mathrm{B}$ signaling pathway in the LPS-priming phase, but independently of the NLRP3 inflammasome activator, nigericin-triggering phase (12).

As mentioned above, EXSs can carry functional proteins and RNAs related to many diseases. Since EXSs represent cell-to-cell communication mediators (13), and their contents are protected from degradation, they are thought to be useful biomarkers considering the inflammasome activation (14). Furthermore, these functional RNAs can be transported from an EXS donor cell to recipient cells, and enable transcriptional changes (15). Although there are extensive studies on biological functions of EXS in inflammatory diseases, mechanistic explanations of how the inflammasome activity regulates secretion of EXS, and how their cargo modulates the biological properties of inflammatory cells are unclear (4). Thus, NLRP3 inflammasome, which is known to be released from damaged cells may contribute to the development of acute graft-versus-host disease via IL-1 $\beta$-dependent mechanism. Increased levels of active caspase- 1 and IL- $1 \beta$ are found in circulating leukocytes and lesions of transplant patients (16). Moreover, nucleic acids containing EXSs-especially miRNAs and long noncoding RNAs (lncRNAs) have been shown to mainly influence insulin signals in target tissues, affecting cell viability, and modulating inflammatory pancreatic cells. In this context, it is thought that the EXS
miRNAs can be used as reliable biomarkers in diabetes (17). Additionally, in diabetes mellitus, it is claimed that the EXSs cargo may signal to transform the endothelial cells from normal phenotype into a diabetic phenotype. However, it is still unclear whether EXS is a precise marker for the detection of obesity related diabetes mellitus (18). Recently, characterization of the circulating exosomal proteins in SARS-CoV-2 infection has revealed the role of macrophage activation syndrome as the main driver of hyperinflammatory response in the mechanisms associated with tissue damage and multiple organ dysfunctions in coronavirus disease-19 (COVID-19) patients (19). On the other hand, tumor-derived EXSs convert macrophages to tumor-promoting type via regulating proteasomal degradation, and activate NLRP3 inflammasome signaling pathway, which promotes lung cancer progression by IL$1 \beta$ secretion (20). However, the potential participation of macrophage derived EXSs to heterotypic cell communication in tumors has been poorly studied.

In brief, EXSs as therapeutic tools for potential use in medicine, have properties for allowing the identification of new biomarkers (21). Actually, atheroprotective Kruppellike factor regulates inflammation-associated miRNA-155 expression in human endothelial cells. When miRNA-155 plus ox-LDL-induced endothelial cells derived EXSs are transferred to human monocytic THP 1 cells, it enhances the shifting of the monocytes/macrophages balance from anti-inflammatory M2 macrophages towards proinflammatory M1 macrophages. This alteration exerts a regulatory effect on NLRP3 inflammasome activation in macrophages. Consequently, EXSs from Kruppel-like factor 2-expressing endothelial cells suppress monocyte activation and diminish proinflammatory response $(22,23)$. Similarly, the proinflammatory M1-like-type macrophages release proinflammatory cargo carrier EXSs after myocardial infarction, thereby myocardial injury is accelerated. These EXSs also contain proinflammatory miRNAs, which are transferred to endothelial cells, leading to the inhibition of angiogenesis and cardiac function by downregulating its novel target genes. Although this mechanism provides a novel insight into the therapeutic approach, how it works still could not be elucidated (24). It is observed that tumorassociated macrophage (TAM)- EXSs comprises signature of 62 proteins. While TAMs are largely immunosuppressive, their EXSs may also have the potential to stimulate, rather than limit, anti-tumor immunity. Therefore, the two faces of TAM-EXS signature can influence the tumor immunity in two opposite directions $(25,26)$. 
These studies suggest that EXS secretion constitutes an essential part of the NLRP3 inflammasome-mediated immune response. Although, it was found that the inflammasomes trigger EXS secretion, the content of exosomal cargo and its case specific properties has remained uncharacterized. In this respect, Budden et al. have characterized the transcriptional cargo of EXSs released by human macrophages upon stimulation with multiple inflammatory triggers, including inflammasome and TLR stimuli (6).

Budden et al. firstly, showed that LPS plus nigericin increased inflammasome responses when transferred to pre-primed macrophages. In contrast, subsequent inflammasome activation was inhibited when LPS plus nigericin or LPS plus R837 (NLRP3 activators) were transferred to unprimed primary macrophages. These data demonstrate that inflammasome-triggered EXSs can modulate the impact of $\mathrm{EXSs}_{\mathrm{NLRP3}}$ on the transcriptome of recipient cells. In other words, $\mathrm{EXS}_{\mathrm{NLRP} 3}$ could either increase or decrease inflammatory responses depending on the recipient cells' priming state and control the severity of inflammation (6).

Inconsistency between the content of inflammasome induced EXSs and their effects on gene expression in recipient cells constitutes a highly important finding. The characterization of EXSs content by type of EXSs and EXS inducing conditions will open a new field of research to uncover the mechanisms behind the function of the EXS.

\section{Acknowledgments}

Funding: None.

\section{Footnote}

Provenance and Peer Review: This article was commissioned by the editorial office, ExRNA. The article has undergone external peer review.

Conflicts of Interest: Both authors have completed the ICMJE uniform disclosure form (available at https://dx.doi. org/10.21037/exrna-21-16). The authors have no conflicts of interest to declare.

Ethical Statement: Both authors are accountable for all aspects of the work in ensuring that questions related to the accuracy or integrity of any part of the work are appropriately investigated and resolved.
Open Access Statement: This is an Open Access article distributed in accordance with the Creative Commons Attribution-NonCommercial-NoDerivs 4.0 International License (CC BY-NC-ND 4.0), which permits the noncommercial replication and distribution of the article with the strict proviso that no changes or edits are made and the original work is properly cited (including links to both the formal publication through the relevant DOI and the license). See: https://creativecommons.org/licenses/by-nc-nd/4.0/.

\section{References}

1. Yang M, Chen J, Su F, et al. Microvesicles secreted by macrophages shuttle invasion-potentiating microRNAs into breast cancer cells. Mol Cancer 2011;10:117.

2. Zhang Y, Liu F, Yuan Y, et al. Inflammasome-Derived Exosomes Activate NF- $\kappa B$ Signaling in Macrophages. J Proteome Res 2017;16:170-8.

3. Hezel MEV, Nieuwland R, Bruggen RV, et al. The Ability of Extracellular Vesicles to Induce a Pro-Inflammatory Host Response. Int J Mol Sci 2017;18:1285.

4. Cypryk W, Nyman TA, Matikainen S. From Inflammasome to Exosome-Does Extracellular Vesicle Secretion Constitute an Inflammasome-Dependent Immune Response? Front Immunol 2018;9:2188.

5. Platnich JM, Muruve DA. NOD-like receptors and inflammasomes: A review of their canonical and noncanonical signaling pathways. Arch Biochem Biophys 2019;670:4-14.

6. Budden CF, Gearing LJ, Kaiser R, et al. Inflammasomeinduced extracellular vesicles harbour distinct RNA signatures and alter bystander macrophage responses. J Extracell Vesicles 2021;10:e12127.

7. Guarda G, Braun M, Staehli F, et al. Type I interferon inhibits interleukin-1 production and inflammasome activation. Immunity 2011;34:213-23.

8. Akira S, Uematsu S, Takeuchi O. Pathogen recognition and innate immunity. Cell 2006;124:783-801.

9. Matikainen S, Nyman TA, Cypryk W. Function and Regulation of Noncanonical Caspase-4/5/11 Inflammasome. J Immunol 2020;204:3063-9.

10. Lamkanfi M, Dixit VM. Mechanisms and functions of inflammasomes. Cell 2014;157:1013-22.

11. Hayden MS, Ghosh S. Shared principles in NF-kappaB signaling. Cell 2008;132:344-62.

12. Wang Z, Maruyama K, Sakisaka Y, et al. Cyclic Stretch Force Induces Periodontal Ligament Cells to Secrete Exosomes That Suppress IL-1 $\beta$ Production Through 
the Inhibition of the NF- $\kappa$ B Signaling Pathway in Macrophages. Front Immunol 2019;10:1310.

13. Yáñez-Mó M, Siljander PR, Andreu Z, et al. Biological properties of extracellular vesicles and their physiological functions. J Extracell Vesicles 2015;4:27066.

14. Yuana Y, Sturk A, Nieuwland R. Extracellular vesicles in physiological and pathological conditions. Blood Rev 2013;27:31-9.

15. Valadi H, Ekström K, Bossios A, et al. Exosome-mediated transfer of mRNAs and microRNAs is a novel mechanism of genetic exchange between cells. Nat Cell Biol 2007;9:654-9.

16. Jankovic D, Ganesan J, Bscheider M, et al. The Nlrp3 inflammasome regulates acute graft-versus-host disease. $\mathrm{J}$ Exp Med 2013;210:1899-910.

17. Chang W, Wang J. Exosomes and Their Noncoding RNA Cargo Are Emerging as New Modulators for Diabetes Mellitus. Cells 2019;8:853.

18. Pardo F, Villalobos-Labra R, Sobrevia B, et al. Extracellular vesicles in obesity and diabetes mellitus. Mol Aspects Med 2018;60:81-91.

19. Barberis E, Vanella VV, Falasca M, et al. Circulating Exosomes Are Strongly Involved in SARS-CoV-2 Infection. Front Mol Biosci 2021;8:632290.

20. Liang M, Chen X, Wang L, et al. Cancer-derived exosomal TRIM59 regulates macrophage NLRP3 inflammasome

doi: 10.21037/exrna-21-16

Cite this article as: Engin A, Engin AB. Why should the molecular characterization of inflammasome-induced exosomal cargo be done? ExRNA 2021;3:6. activation to promote lung cancer progression. J Exp Clin Cancer Res 2020;39:176.

21. Panfoli I, Santucci L, Bruschi M, et al. Microvesicles as promising biological tools for diagnosis and therapy. Expert Rev Proteomics 2018;15:801-8.

22. He S, Wu C, Xiao J, et al. Endothelial extracellular vesicles modulate the macrophage phenotype: Potential implications in atherosclerosis. Scand J Immunol 2018;87:e12648.

23. Yin R, Zhu X, Wang J, et al. MicroRNA-155 promotes the ox-LDL-induced activation of NLRP3 inflammasomes via the ERK1/2 pathway in THP-1 macrophages and aggravates atherosclerosis in ApoE-/- mice. Ann Palliat Med 2019;8:676-89.

24. Liu S, Chen J, Shi J, et al. M1-like macrophage-derived exosomes suppress angiogenesis and exacerbate cardiac dysfunction in a myocardial infarction microenvironment. Basic Res Cardiol 2020;115:22.

25. Hoves S, Ooi CH, Wolter C, et al. Rapid activation of tumor-associated macrophages boosts preexisting tumor immunity. J Exp Med 2018;215:859-76.

26. Cianciaruso C, Beltraminelli T, Duval F, et al. Molecular Profiling and Functional Analysis of Macrophage-Derived Tumor Extracellular Vesicles. Cell Rep 2019;27:30623080.e11. 